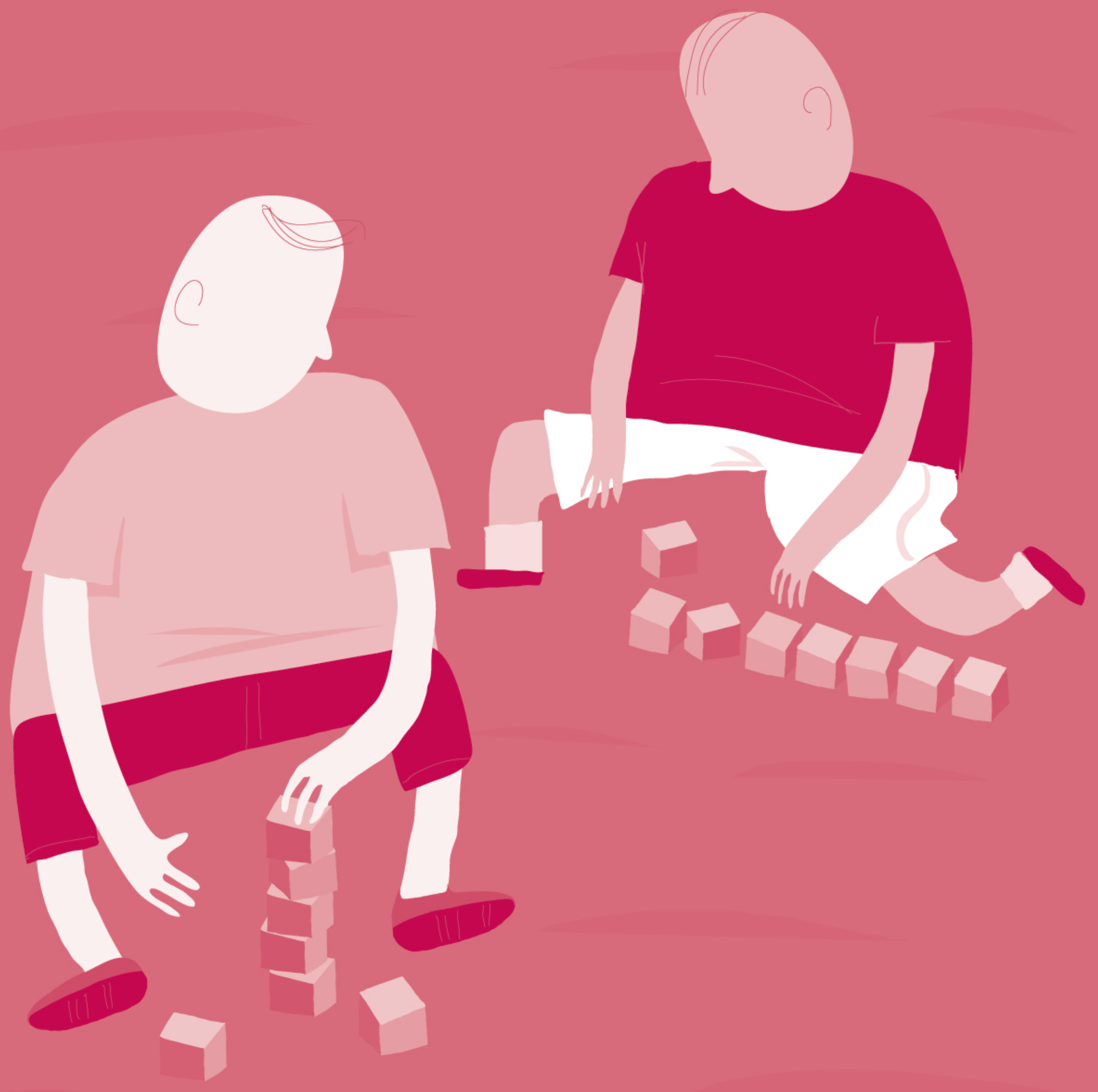




\section{Diferencias en el estilo de comunicación entre empleados finlandeses y mexicanos cuando se comunican con sus jefes: un estudio comparativo}

Federico Varona

- Doctor y maestro en Estudios de la Comunicación por la Universidad de Kansas, EE. UU.

- Licenciado en Teología (España)

- Diplomado en Psicología (España) y Comunicación Audiovisual (Francia)

- Profesor del Departamento de Estudios de Comunicación de la San José State University, EE. UU.

- Miembro del Consejo Editorial de Organicom

- www.sjsu.edu/faculty/fvarona

- fvarona@sjsu.edu

Päivi Vaahterikko-Mejía, PhD

- Doctora en Humanidades por la Universidad de Jyváskylá, Finlandia

- Profesora del Departamento de Comunicación, Comunicación Organizacional y Relaciones Públicas de la Universidad de Jyváskylá, Finlandia

- paivi.vaahterikko-mejia@jyu.fi

Mariela Pérez Chavarría

- Maestra en Ciencias con especialidad en Comunicación por el Tecnológico de Monterrey, Campus Monterrey, México

- Maestra en Formación y Capacitación de Recursos Humanos por la Universidad Autónoma de Nuevo León (UANL), México

- Licenciada en Ciencias de la Comunicación por la Universidad Regiomontana, México

- Licenciada en Letras Españolas por el Tecnológico de Monterrey, Campus Monterrey

- Profesora titular del Departamento de Comunicación del Campus Monterrey

- mariela.perez@itesm.mx 
O objetivo deste artigo é apresentar os resultados do estudo realizado por uma equipe internacional de pesquisadores interessados em descobrir e comparar os estilos de comunicação de funcionários quando interagem com seus superiores, na Finlândia e no México. Para tanto, apresentamos, em primeiro lugar, um marco teórico do estudo; em segundo, a metodologia; em terceiro, os resultados da análise estatística comparativa entre Finlândia e do México; em quarto, as conclusões gerais e sua explicação cultural; e, em quinto, as implicações teóricas e práticas deste estudo para a comunicação funcionário/superior nas organizações.

PALAVRAS-CHAVE: COMUNICAÇÃO ORGANIZACIONAL • ESTILOS DE COMUNICAÇÃO • RELAÇÃO DE HIERARQUIA • FINLÂNDIA • MÉXICO

\section{Abstract}

The purpose of this article is of presenting the results of the study conducted by a team of international researchers interested in discovering and comparing the communication styles of employees when interacting with their superiors, in Finland and in Mexico. Thus, we first present a theoretical mark of the study; second, the methodology; third, the results of the comparative statistical analysis between Finland and Mexico; fourth, the general conclusions and their cultural explanation; and fifth, the theoretical and practical implications of the study for employee-superior communication in the organizations.

KEYWORDS: ORGANIZATION COMMUNICATION - COMMUNICATION STYLES - HIERARCHICAL RELATIONSHIP • FINLAND • MEXICO

\section{Resumen}

El objetivo es presentar los resultados del estudio realizado por un equipo internacional de investigadores interesados en descubrir y comparar los estilos de comunicación de los empleados cuando interactúan con sus superiores, en Finlandia y México. Para ello, presentamos: primero, un marco teórico del estudio; segundo, la metodología; tercero, los resultados del análisis estadístico comparativo entre Finlandia y México; cuarto, las conclusiones generales y su explicación cultural; y quinto, las implicaciones teóricas y prácticas de este estudio con respecto a la comunicación empleado/superior en las organizaciones.

PALABRAS CLAVE: COMUNICACIÓN ORGANIZACIONAL • ESTILOS DE COMUNICACIÓN • RELACIÓN DE JERARQUÍA • FINLANDIA • MÉXICO 


\section{Marco Teórico}

L a comunicación efectiva en el trabajo es uno de los principales retos que actualmente enfrentan las empresas y organizaciones multiculturales, tanto a nivel nacional como internacional (SHUTER \& WISEMAN, 1994; TEBOUL, CHEN \& FRITZ, 1994; KREPS \& KUNIMOTO, 1994; COX, 1993; HARRIS \& MORAN, 1987). La nueva tecnología y la internacionalización del contexto organizacional traen diferentes desafíos como también necesidades para las empresas y organizaciones. La calidad de la comunicación superior/empleado es clave para el buen funcionamiento de una empresa u organización.

\section{Estilo de comunicación y diferencias culturales}

Una de las primeras propuestas tendientes a definir qué se entiende por estilo de comunicación es el realizado por Norton en 1983. Según este autor el estilo de comunicación es la manera como los individuos se comunican entre sí verbal y no verbalmente. Las características fundamentales de estilo de comunicación, según Norton, son: el ser observable, polifacético, y que permite saber como debe ser tomado, interpretado, analizado, y entendido el sentido literal del mensaje. Para este autor, las dimensiones del estilo de comunicación son: atento, relajado, activo (no verbal), dominante, argumentador, capaz de dejar impresión, o combinaciones de estos como atento y comprensivo, activo y dominante (NORTON, 1983).

Hasta la fecha son varios los estudios que se han realizado para definir el perfil o estilo de comunicación de las diferentes culturas o nacionalidades y explicar el impacto que la cultura tiene en él. Por ejemplo, se han realizado varios trabajos para demostrar cómo los modos de comunicación de las culturas de Asia del Este están influenciados por las filosofias confucionistas (YUM, 1988). Uno de los principios de esta filosofía $\mathrm{Li}$ (propiedad, formalidad en la relación) estipula que en las relaciones interpersonales en sus diferentes niveles, el individuo debe comportarse correctamente de acuerdo con las expectaciones de su papel o posición. Chen y Chung (1994) resaltan cómo en la relación superior-subordinado, el superior está obligado a proteger y preocuparse de todas las necesidades del subordinado. Por su parte, el subordinado está obligado a demostrar respeto, lealtad, compromiso a su superior.

Condon (1985) es uno de los primeros investigadores que trata de entender y explicar cómo las diferencias culturales que existen entre México y Estados Unidos han influi- 
do en el estilo de comunicación de cada país y cómo lograr una comunicación competente o efectiva. Otra investigadora que ha estudiado las diferencias culturales entre México y Estados Unidos y cómo influyen en el estilo de gerencia y de comunicación en las empresas de cada país es Kras (1988). Y más recientemente Aragon y Phillips' (1995) realizaron un estudio sobre las habilidades comunicativas de líderes hispanos y descubrieron que la mayoría de los líderes entrevistados creían que debido a sus características culturales las habilidades comunicativas de sus colegas euroamericanos debían ser diferentes.

En otro estudio realizado por Varona (1995), para investigar similitudes y diferencias entre empleados hispanos y euroamericanos en el estilo comunicativo cuando se comunican con sus superiores, descubrió que efectivamente existieron diferencias estadísticamente significativas en algunas conductas comunicativas entre ambos grupos. Al mismo tiempo el autor demuestra como ciertas diferencias culturales y el proceso de aculturación realizado por los empleados hispanos explican algunas de las diferencias y similitudes comunicativas entre empleados hispanos y euroamericanos, y entre hispanos nacidos en Estados Unidos e hispanos nacidos en países latinoamericanos.

\section{Cultura nacional y cultura organizacional en Finlandia}

La cultura nacional alude a formas de pensar, de hablar, y de comprender el mundo en una comunidad o en un grupo que comparte un mismo sistema legal y de gobierno que regula su conducta social y política. Vaahterikko-Mejía (2001, p. 24) define la cultura nacional como

"el capital social de una comunidad que vive dentro de un área fisica delimitada por fronteras geográficas legales y fronteras mentales, y que comparten, entre otros elementos, el símbolo de una bandera nacional, una divisa legalmente aceptada, una o varias religiones e idiomas, y sistema común de gobierno".

Las dimensiones culturales de una nación reflejan las tendencias que caracterizan el comportamiento de la mayoría de la población. Las tendencias más características de la cultura nacional finlandesa son las siguientes: Primera, el universalismo: las normas y leyes se aplican por igual a todas las personas sin importar las relación que se tiene con ellas. Segunda, el individualismo: se aprecian logros individuales y la capacidad de tomar iniciativas individuales. Tercera, la neutralidad emocional: en Finlandia no se suele mostrar las emociones muy abiertamente ni en las conversaciones superficiales. Hay fuertes emociones obviamente, pero se muestran con las personas de mucha confianza. Hay que notar que las personas de confianza no son solamente los miembros de la familia, sino también colegas y amigos siempre cuando se ha detectado un grado importante de confianza con ellos. Cuarta, la comunicación es específica: en las relaciones profesionales, el énfasis de la comunicación y negociación se pone en lo que es el objetivo de la misma y no tanto en las relaciones humanas. Quinta, se enfatizan los lo- 
gros individuales: en Finlandia las personas se evalúan según sus logros individuales sin tomar tanto en cuenta el trasfondo de los mismos, como podría ser los logros familiares. Sexta, la orientación es hacía el futuro: por consiguiente se trata de planear lo más posible para evitar errores y situaciones de ambigüedad. Séptima, la actitud hacia el entorno es de dominio: en Finlandia, el individuo cree que puede modificar su entorno. Octava, la distancia de poder es baja: la estructura jerárquica en Finlandia es limitada y no es objeto de culto como en otras culturas. Novena, se enfatiza la feminidad: en Finlandia los roles de sexo no son muy marcados, existe una fuerte tendencia a cuidar a los que están más incapacitados y necesitados y se tiene mucha empatía hacia los débiles. Décima, la tolerancia hacia la incertidumbre es alta: se puede decir que en Finlandia se tiene cierta tolerancia hacia la inseguridad (según Hofstede, Finlandia se sitúa entre los países con un índice negativo en esta categoría (-27) lo que significa que existe tolerancia hacia la incertidumbre aunque no es muy alta (HOFSTEDE, 1991). Efectivamente, en Finlandia existe también un fuerte sentido de la responsabilidad y tendencia a seguir las normas de la sociedad. Undécima, la comunicación es explícita (el contexto es bajo): se dice o se escribe directamente lo que se quiere comunicar. Los mensajes son directos y explícitos, las reacciones son más abiertas y fáciles de observar, se enfatiza la comunicación verbal, y el sentido y uso del tiempo es muy estructurado. Duodécima, se enfatiza la cortesía negativa: en Finlandia se tiende a minimizar las cualidades y logros de la personas. Existe cierta dosis de ironía y algunas veces hasta cinismo al hacer "las alabanzas". Sin embargo, se trata de una forma de cortesía con un cierto enfoque positivo ya que se trata de no querer molestar a la otra persona y de respeta mucho la independencia del individuo. Decimotercera, el concepto de tiempo es monocrónico con respecto a lo que se hace: cumplir primero con lo que se ha comprometido o concluir lo que se está haciendo es muy importante en Finlandia antes de comprometer a algo nuevo o iniciar nuevos proyectos. Es importante ser puntual y no malgastar el tiempo.

Otras características específicas de la cultura organizacional finlandesa son las siguientes según Vaahterikko-Mejía (2001): la toma de decisión se hace democráticamente respetando y escuchando la opinión de todo el grupo. Esto, aunque puede afectar la demora del proceso, es muy importante ya que como se dijo anteriormente y se debe en parte al bajo índice en la distancia del poder y lo limitado de la estructura jerárquica. Las decisiones y acuerdos se hacen con seriedad y su cumplimiento se efectúa con puntualidad. La buena organización en la empresa es altamente valorada y la planificación se hace con mucha anticipación. Por otra parte, la planeación es minuciosa ya que existe un temor muy alto a los cambios y errores. Esto a su vez ocasiona que se dé cierta rigidez en el proceso de planificación. Las normas se respetan mucho, aunque sean pocas, para asegurar que las cosas funcionen con normalidad y garantizar la satisfacción de todos. La palabra dada y los compromisos se toman muy en serio. Finalmente se trata de ser muy práctico en la manera de proceder en todos los asuntos relacionados con el funcionamiento de la empresa. 
DIFERENCIAS EN EL ESTILO DE COMUNICACIÓN ENTRE EMPLEADOS FINLANDESES Y MEXICANOS CUANDO SE COMUNICAN CON SUS JEFES: UN ESTUDIO COMPARATIVO - FEDERICO VARONA, PÄIVI VAAHTERIKKO-MEJÍA E MARIELA PÉREZ CHAVARRÍA

\section{Cultura nacional y cultura organizacional en México}

En México, como en cualquier otro lugar del mundo, la cultura de las organizaciones se ve afectada por las variables propias de la cultura nacional y hasta la regional. Así por ejemplo, en el caso de la institución en donde se recogieron los datos para esta investigación, su cultura concuerda con ciertos valores de la zona norte del país, caracterizada por el alto aprecio al trabajo como actividad esencial humana (PALACIOS, 1999). También existe alto compromiso por el cumplimiento y la eficiencia. De la Cerda y Núñez (1993), de acuerdo con las categorías de Hofstede, identifican en general cuatro características distintivas de la cultura mexicana que conforman el contexto o marco referencial básico en el que se inserta la cultura de las organizaciones nacionales. Obviamente hay diferentes tipos de organizaciones, pero de manera global en ellas puede encontrarse: (1) Alto indice de distancia de poder, lo cual repercute en el tipo de organizaciones muy centralizadas y en las que predomina un liderazgo autocrático y paternalista. (2) Alto indice de temor ante la incertidumbre. Esto explica la preferencia por gran cantidad de reglas definidas que determinan las conductas esperadas y delimitan en general las condiciones de trabajo. (3) Bajo indice de individualismo y por lo tanto, alto en colectivismo. Para los investigadores esta característica explica el que las personas se comprometan más con la organización porque valoran las relaciones y la seguridad. (4) Alto indice de masculinidad, debido - entre otras causas - a que la debilidad y la feminidad son signos culturales que reflejan sometimiento o devaluación social en la cultura mexicana. Por lo tanto se valora más la fortaleza y la seguridad en general.

Nicholls, Lane y Brehem (1999) en un estudio acerca de equipos de trabajo auto administrados en México, también coinciden con lo antes expuesto y refieren, por ejemplo, que el marcado paternalismo se evidencia en que los administradores toman las decisiones, establecen objetivos y resuelven conflictos, mientras que los trabajadores suelen esperar instrucciones y las decisiones que toman van de acuerdo con su rol y su nivel. Con esta tradición, en las organizaciones se evita la ambigüedad porque se sabe de antemano dónde reside el poder y quien fija las reglas, impone castigos y premia. En su investigación, Nicholls et al. (1999) hablan de una tradición que rinde culto al jefe, lo cual es cierto porque tanto en las organizaciones como en la vida social, la cultura mexicana se caracteriza porque tiende a ser muy jerárquica y con alta distancia de poder. Sin dudad esta conducta es el reflejo de la propia historia del país en donde por tradición la autoridad reside en lo más alto de la jerarquía y al jefe (sea el padre, el presidente o gobernante, o el director) siempre se le da un estatus elevado con altos poderes para sancionar (cultura muy vertical), pero también con la responsabilidad de brindar protección.

Al respecto, Martínez y Dorfman (1998) agregan que quizá por influencia de la familia (valor fundamental en la cultura mexicana) este modelo donde el padre protege y decide, también está presente en las organizaciones mexicanas. Se trata de un mode- 
lo de organización patriarcal en donde el jefe o dueño brindan seguridad y protección a sus empleados a cambio de lealtad. El sistema familiar, dicen estos investigadores, sirve como modelo a las organizaciones y permite entender los comportamientos de los jefes con los subordinados, particularmente la importancia que se le da a la confianza y la solidaridad. Otro hecho que destacan Nicholls et al. (1999) es que en los trabajadores mexicanos los valores colectivistas a menudo son trasladados al deseo de buscar la armonía en el lugar de trabajo, con lo que se evita el conflicto. Por ello - dicen estos investigadores - los trabajadores mexicanos han desarrollado habilidades de trabajo en grupo y cooperación.

Si toman algunas de las variables de Trompenaars y Hampden-Turner (1998) mencionadas anteriormente, de manera global puede decirse que las tendencias más características de la cultura mexicana son: Primera: particularismo. Las leyes y normas varían según la relación y situación que se tiene con las personas en cuestión, aunque se tiende a ser objetivos. Segunda: hay orientación hacia las personas. Tercera: en negociaciones se tiende a ser específico para concentrarse en el negocio, pero no es extraño que puedan convivir aspectos difusos; es decir, que se interrelacionen aspectos de la vida personal y laboral. Cuarta: el concepto de tiempo tiende a la policronía; es decir, las personas suelen estar involucradas en varios proyectos a la vez y hacer más de dos cosas. Saben que tarde o temprano acabarán todo y a medida que terminan algo surgen más proyectos nuevos en los que se van involucrando. Son flexibles con los horarios de las citas. Quinta: en México el contexto es alto; sobre todo en la comunicación verbal se dan por supuestas y conocidas muchas cosas y aún en el los mensajes escritos, aunque se tiende a ser explícito y concreto, puede omitirse información de manera involuntaria porque se da por sabida.

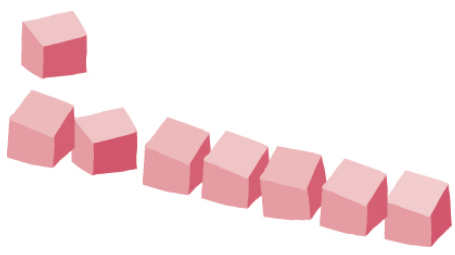


DIFERENCIAS EN EL ESTILO DE COMUNICACIÓN ENTRE EMPLEADOS FINLANDESES Y MEXICANOS CUANDO SE COMUNICAN CON SUS JEFES: UN ESTUDIO COMPARATIVO - FEDERICO VARONA, PÄIVI VAAHTERIKKO-MEJÍA E MARIELA PÉREZ CHAVARRÍA

\section{Metodología}

En esta sección presentamos una breve descripción: primero, del cuestionario usado para la recogida de información; segundo, de las muestras de la investigación (las organizaciones de Finlandia y México); y tercero, del análisis estadístico de la información.

\section{Cuestionario de Estilo de Comunicación Empleado-Superior (CECES)}

El instrumento usado en esta investigación para recoger información fue el Cuestionario del Perfil de Conductas Comunicativas Empleado-Superior (CPCCES) (Copyright, 1995-2005) de Federico Varona. Este cuestionario fue creado para evaluar la frecuencia de conductas comunicativas que los empleados usan cuando se comunican con sus superiores. El cuestionario está integrado por una batería de sesenta y ocho preguntas y usa una escala de respuestas que va de uno a cinco, donde 1 = "nunca"; 2 = "raras veces"; 3 = "algunas veces"; 4 = "muchas veces"; y 5 = "siempre". El cuestionario contiene catorce dimensiones de cinco preguntas cada una, excepto la última que es de tres. Estas son las dimensiones: 1) Habilidades para escuchar (01-05); 2. Comunicación no verbal $(06-10) ; 3$. Comunicación verbal $(11-15) ; 4$. Comunicación formal/informal (16 - 20); 5. Contenido de la comunicación $(21-25) ; 6$. Comunicación sobre el trabajo (26-30); 7. Feedback recibido $(31-35) ; 8$. Apertura en la comunicación (3640); 9. Canales de comunicación (41-45); 10. Manejo del conflicto comunicacional $(46-50) ; 11$. Feedback dado $(51-55) ; 12$. Toma de decisiones y comunicación (56$60) ; 13$. Demográficos y comunicación (61 - 65); 14. Impacto de la comunicación (6668). La última sección del cuestionario contiene preguntas demográficas referentes al sexo, la cultura, y la edad del empleado y del superior, y al tipo de empresa u organización en la que trabaja el empleado.

La validez del cuestionario con respecto a su contenido, al concepto que mide, y a su aparente evidencia está claramente demostrada al hacer un análisis del contenido de las preguntas y de sus dimensiones (KRATHWOHL, 1993). Por otra parte, la confiabilidad de la consistencia interna y estabilidad del cuestionario fue de (.70) cuanto se realizó el análisis de Cronbach Alphas en el estudio piloto que se hizo del cuestionario (VARONA, 1995) lo cual se considera un índice aceptable. El índice de confiabilidad del cuestionario en las muestras de esta investigación fue de (.76) en la de Finlandia y de (.79) en la de México (SPSS Reliability Test, 2004) lo que demuestra de la consistencia interna y estabilidad del cuestionario.

\section{Las muestras de la investigación}

Las muestras usadas para esta investigación fueron recogidas en dos países, Finlandia y México. En Finlandia la muestra se recogió en tres empresas diferentes y en México en una universidad privada del norte del país. 
DIFERENCIAS EN EL ESTILO DE COMUNICACIÓN ENTRE EMPLEADOS FINLANDESES Y MEXICANOS CUANDO SE COMUNICAN CON SUS JEFES: UN ESTUDIO COMPARATIVO - FEDERICO VARONA, PÄIVI VAAHTERIKKO-MEJÍA E MARIELA PÉREZ CHAVARRÍA

\section{Muestra de Finlandia}

Para recoger la información en Finlandia primero se tuvo que hacer la traducción del Cuestionario Estilo de Comunicación Empleado-Superior (Federico Varona, Ph. D.: Copyright, 1995-2007) al finlandés. Esta traducción se hizo desde la versión inglesa, de acuerdo con las normas internacionales usadas en la investigación intercultural (HOFSTEDE, 1980).

La muestra de Finlandia consistió de 109 (N 109) empleados (todos finlandeses), de los cuales 50 (46\%) fueron hombres y 58 (54\%) mujeres. Las características de los superiores con que se comunicaron estos empleados fueron las siguientes: En la muestra de Finlandia todos los superiores (supervisores) eran finlandeses de los cuales 99 (93\%) eran hombres y 7 (7\%) mujeres. El cuanto al tipo de empresa u organización en la que trabajaban los empleados de Finlandia, 55 empleados (59\%) trabajaban en fábricas de producción; 27 en departamentos de ventas (29\%); y 11 en la Bolsa (12\%).

La primera empresa donde se recogieron los datos para esta investigación fue uno de los mayores almacenes que existen en el área central de Finlandia. En el año 2003 el $59 \%$ de las familias de la región pertenecían a este sector. La empresa tiene también servicios hoteleros y de restaurantes. Los propietarios de la empresa son sus 75000 clientes los cuales tienen participación en la dirección de la empresas a través del voto. La empresa tiene 89 sedes, en las cuales trabajan más de 1500 personas. La segunda empresa donde se recogió información para este estudio fue una Fábrica productora de papel y de las máquinas de láminas de papel situada en una ciudad del norte de Finlandia, pero con varias fábricas y sedes en Finlandia y en el exterior. La tercera empresa fue es el líder internacional manufacturero y de la tecnología diversificada, sirviendo clientes mundialmente con productos y servicios aerospaciales, tecnología de control para las construcciones, y productos de automóviles. Una característica común y fundamental de la cultura de estas empresas es la participación democrática de los empleados en la toma de decisiones.

\section{Muestra de México}

Para recoger la información en México se uso la versión española del Cuestionario Estilo de Comunicación Empleado-Superior (Federico Varona, Ph.D.: Copyright, 1995-2004).

Para definir la muestra, del total de empleados (284) de la sección elegida, se eliminaron los extranjeros (casi un $20 \%$ de la población) ya que se trataba de conocer el estilo de comunicación de empleados mexicanos. Así, con la población restante se realizó un muestreo aleatorio estratificado, con un nivel de confianza del $90 \%$, encuestando a un total de 107 empleados, un 37,67\% de la población total. La muestra se dividió en los siguientes estratos: por departamento (de Comunicación, de Relaciones Internacionales, de Lenguas Modernas y de Estudios Humanísticos), y por puesto del 
empleado (maestros de planta, maestros de cátedra, administrativos de plata y administrativos eventuales).

La composición final de la muestra quedó como se describe a continuación: sexo: 82 empleados fueron mujeres (77\%) y 25 hombres (23\%). Nivel de educación: secundaria: $1(1 \%)$; preparatoria: 13 (12\%); profesional: $16(15 \%)$; y postgrado: $77(72 \%)$. Edad: de 18 a 25 años: 14 (13\%); de 26 a 35 años: 34 (32\%); y más de 36 años: 59 (55\%). Sexo del jefe inmediato: femenino: 84 (78\%); masculino: 23 (22\%). Departamento al que pertenecen: Comunicación: 29 (27\%); Relaciones Internacionales: 24 (22\%); Lenguas Modernas: 38 (36\%); Estudios Humanísticos: 16 (15\%). Posición en el trabajo: profesor de cátedra: 36 (34\%); profesor de planta: 37 (35\%); director de departamento: 16 $(15 \%)$; secretaria u otro puesto administrativo de apoyo como mensajero, encargado de almacén, entre otros: $18(16 \%)$.

Los datos se recogieron en una universidad privada, con más de 60 años de antigüedad, y fundada por un grupo de empresarios de una de las ciudades más importantes del norte de México. Actualmente tiene presencia en toda la República Mexicana, Latinoamérica y el mundo a través de su universidad virtual. En la actualidad cuenta con 33 campi, y sedes en México y Latinoamérica. Su población estudiantil total es de casi 95 mil estudiantes, en todos los campi y su planta de profesores global asciende a casi 8 mil, más 14.692 empleados administrativos.

\section{Análisis estadístico de la información}

Los cuestionarios de ambas muestras fueron analizados usando el programa SPSS para Windows versión 11.5.0. Los análisis estadísticos realizados fueron los siguientes: primero se calcularon estadísticas descriptivas (media y la desviación estándar) para cada una de las muestras (Finlandia y México). Segundo, se realizó el Indepent Samples T-test para comparar las medias de cada pregunta de las dos muestras. Tercero, también se calcularon las medias y desviaciones estándares de cada una de las 14 dimensiones del cuestionario para cada una de las muestras (Finlandia y México).

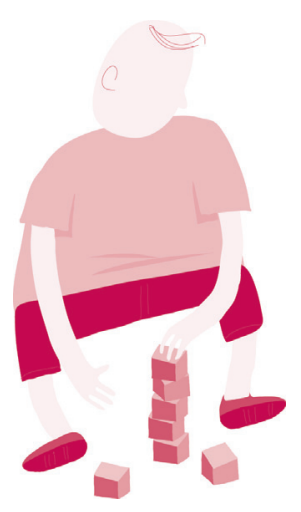




\section{Resultados por dimensiones}

En esta gráfica se presentan las medias de cada una de las 14 dimensiones del Cuestionario de Estilo de Comunicación Empleado-Superior (CECES) correspondientes a los empleados finlandeses y mexicanos. La primera columna (color azul) de cada dimensión representa la media de los empleados finlandeses y la segunda columna (color rojo) la de los empleados mexicanos.

\section{Comparacion por dimensiones}

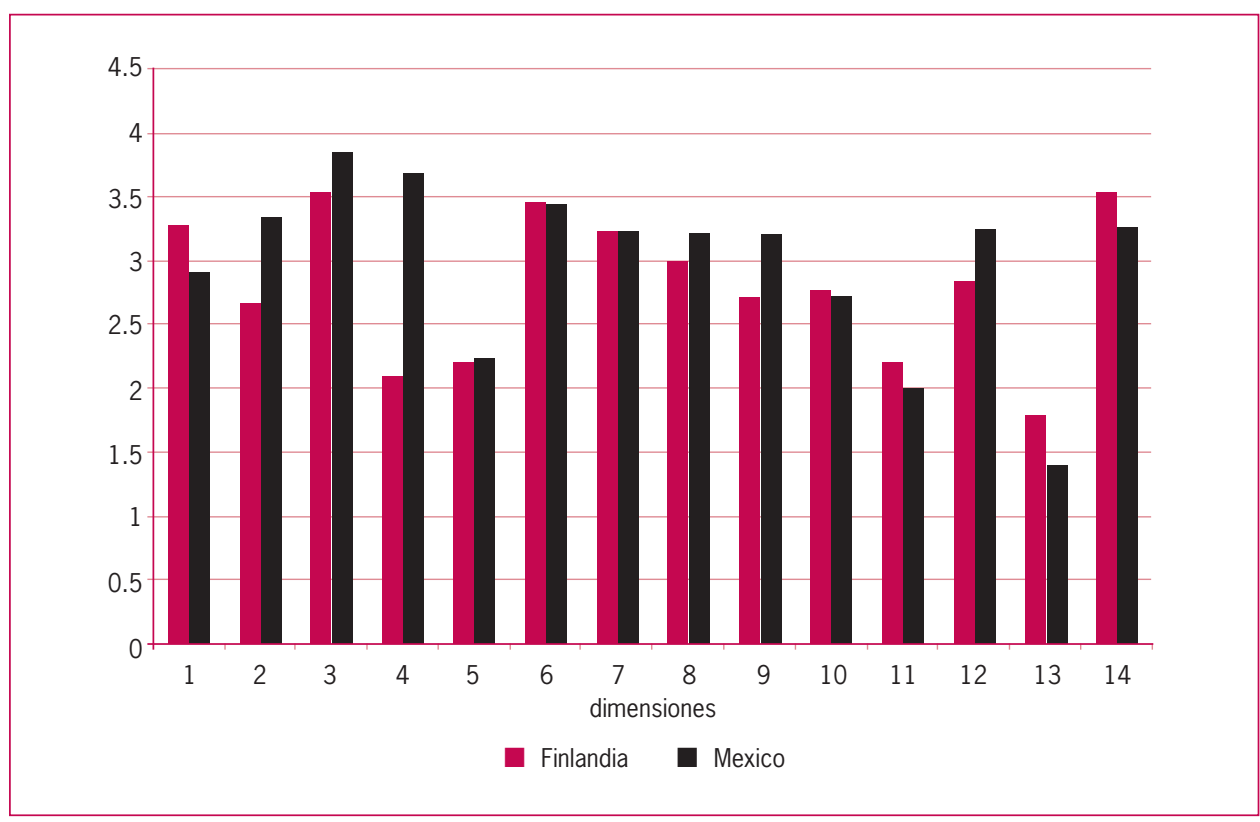

A continuación presentamos los resultados, en forma descriptiva, de las diferencias significativas en el estilo de comunicación entre empleados finlandeses y mexicanos cuando se comunican con sus jefes. Estos resultados están basados exclusivamente en las medias de cada una de las 14 dimensiones que fueron estadísticamente significativas después de aplicar el Indepent Samples T-test. Por razones de espacio no incluimos los resultados numéricos estadísticos. El lector puede solicitarlos dirigiéndose al primer autor de este artículo Dr. Federico Varona a esta dirección: fvarona@sjsu.edu.

\section{Dimensión 1: habilidades para escuchar}

En general, los empleados finlandeses exhibieron más frecuencia de conductas de escucha cuando se comunican con sus jefes que los empleados mexicanos. Específicamente, los empleados finlandeses cuando escuchan a sus jefes interrumpen más frecuente- 
mente, hacen más preguntas para aclarar la información recibida, y repiten mucho más lo que les dicen sus jefes que los empleados mexicanos.

\section{Dimensión 2: comunicación no verbal}

En general, los empleados mexicanos exhibieron más conductas no verbales al comunicarse con sus jefes que los empleados finlandeses. Específicamente, los empleados mexicanos usan más las manos, ven más directamente a los ojos, tocan más frecuentemente en el brazo o hombro, y ponen más atención a cómo dicen las cosas (tono de voz, volumen, velocidad) cuando se comunican con sus jefes que los empleados finlandeses.

\section{Dimensión 3: comunicación verbal}

En general, los empleados mexicanos exhibieron más conductas verbales al comunicarse con sus jefes que los empleados finlandeses. Específicamente, los empleados mexicanos buscan más cuidadosamente sus palabras al expresar sus ideas y son más corteses y correctos cuando se dirigen a sus jefes que los empleados finlandeses.

\section{Dimensión 4: comunicación formal/informal}

En general, los empleados mexicanos exhibieron más conductas de comunicación formal-informal al comunicarse con sus jefes que los finlandeses. Específicamente, los empleados mexicanos cuando se dirigen a sus jefes usan más títulos tales como Señor/a, Lic. /a, Dr. /a; toman con más frecuencia la iniciativa para comunicarse informalmente; y quieren que sus jefes los traten con más formalidad que los empleados finlandeses. Las diferencias entre los dos grupos están claramente marcadas.

\section{Dimensión 5: contenido de la comunicación}

Los empleados mexicanos hablan con más frecuencia de política y de religión con sus jefes que los empleados finlandeses. Por el contrario, los empleados finlandeses hablan con más frecuencia de deportes y diversiones con sus jefes que los mexicanos.

\section{Dimensión 6: comunicación relacionada con el trabajo}

Los empleados mexicanos entienden con más frecuencia las instrucciones de trabajo que les dan sus jefes y aceptan con más frecuencia nuevas ideas sugeridas por sus jefes que los empleados finlandeses. Por el contrario, los empleados finlandeses muestran más abiertamente el desacuerdo con sus jefes en tema relacionados con el trabajo que los empleados mexicanos.

\section{Dimensión 7: recibir feedback}

Los empleados mexicanos desean recibir con más frecuencia comentarios positivos de sus jefes, aceptan con más frecuencia la crítica constructiva, y piden con más frecuencia disculpas a sus jefes cuando hacen algo mal que los empleados finlandeses, Por el contrario, los empleados finlandeses buscan excusas con más frecuencia para 
justificar sus errores y aceptan con más frecuencia que sus jefes les critiquen enfrente de otros en el trabajo que los empleados mexicanos.

\section{Dimensión 8: apertura comunicativa}

En general, los empleados mexicanos exhibieron más conductas de apertura comunicativa al interactuar con sus jefes que los finlandeses. Específicamente, los empleados mexicanos expresan su punto de vista, sus necesidades, y sus expectativas; y confían en sus jefes con más frecuencia que los empleados finlandeses. Por el contrario, los empleados finlandeses utilizan amenazas cuando se comunican con sus jefes con más frecuencia que los empleados mexicanos.

\section{Dimensión 9: canales de comunicación}

En general, los empleados mexicanos hicieron más uso de los canales de comunicación al interactuar con sus jefes que los finlandeses. Específicamente, los empleados mexicanos usaron con más frecuencia que los finlandeses los siguientes canales de comunicación: a) la comunicación cara a cara, b) la comunicación por escrito, y c) el orden jerárquico cuando tienen que comunicar asuntos relativos al trabajo.

\section{Dimensión 10: comunicación y conflicto}

Los empleados finlandeses, cuando se comunican con sus jefes tienen más conflictos en la comunicación pero ceden para resolverlos de manera que sean beneficiosos para ambos. Esto ocurre con más frecuencia que en los empleados mexicanos. Por su parte, los empleados mexicanos cuando se comunican con sus jefes tratan de evitar conflictos con más frecuencia que los empleados finlandeses.

\section{Dimensión 11: dar feedback}

En general, los empleados finlandeses exhibieron más frecuencia de conductas comunicativas relacionadas con el dar feedback al comunicarse con sus jefes que los empleados mexicanos. Específicamente, los empleados finlandeses: a) se sienten con más frecuencia incómodos cuando hacen comentarios positivos a sus jefes; b) hacen con más frecuencia comentarios negativos a sus jefes; c) critican con más frecuencia a sus compañeros de trabajo; y d) hacen comentarios relativos al trabajo a alguien que está en una posición superior a la de sus jefes con más frecuencia que lo empleados mexicanos. Por el contrario, los empleados mexicanos hacen con más frecuencia comentarios positivos a sus jefes que los empleados finlandeses.

\section{Dimensión 12: comunicación y toma de decisiones}

En general, los empleados mexicanos exhibieron más frecuencia de conductas comunicativas relacionadas con la toma de decisiones al comunicarse con sus jefes que los finlandeses. Específicamente, los empleados mexicanos: a) quieren participar en la toma de decisiones con sus jefes, c) defienden sus ideas cuando participan en la toma de decisiones, y c) consultan con sus jefes antes de tomar decisiones con más frecuencia que los empleados finlandeses. Por el contrario, los empleados finlandeses 


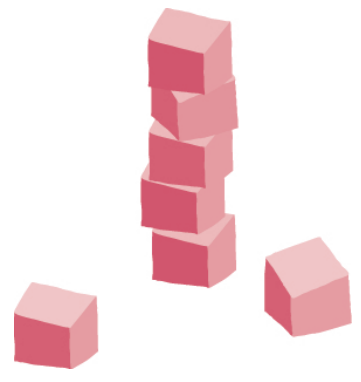

prefieren hacer lo que proponen sus jefes con más frecuencia que los empleados mexicanos.

\section{Dimensión 13: características culturales y comunicación}

En general, los empleados finlandeses cambiaron con más frecuencia su estilo de comunicación dependiendo de las características culturales de sus jefes, que los empleados mexicanos. Específicamente, para los finlandeses el acento de sus jefes constituye un problema para comunicarse con ellos, y el sexo y la edad de sus jefes les hacen cambiar su estilo de comunicación con más frecuencia que a los empleados mexicanos.

\section{Dimensión 14: impacto de la comunicación}

Para los empleados finlandeses, el impacto que tiene la calidad de comunicación cuando se comunican con sus jefes en su nivel de satisfacción en el trabajo es más frecuente ("muchas veces") que en los mexicanos ("algunas veces"). Sin embargo, no existe diferencia entre empleados mexicanos y finlandeses en cuanto al impacto que tiene la calidad de comunicación con sus jefes en la ejecución de su trabajo y el compromiso con la organización. Ambos grupos reconocen que influye "algunas veces".

Las conclusiones presentadas en esta sección claramente demuestran que los estilos de comunicación entre empleados y jefes son diferentes en Finlandia y en México.

\section{Conclusiones generales y explicación cultural}

Las conclusiones generales que presentamos en esta sección sobre las diferencias en el estilo de comunicación entre empleados finlandeses y mexicanos cuando se comunican con sus jefes están basadas exclusivamente en los resultados estadísticamente significativos. Por otra parte, la explicación cultural del porqué de esas diferencias comunicativas la hacemos basados en el análisis de las características culturales nacionales y organizacionales de los respectivos países, es decir, de Finlandia y México. 
DIFERENCIAS EN EL ESTILO DE COMUNICACIÓN ENTRE EMPLEADOS FINLANDESES Y MEXICANOS CUANDO SE COMUNICAN CON SUS JEFES: UN ESTUDIO COMPARATIVO - FEDERICO VARONA, PÄIVI VAAHTERIKKO-MEJÍA E MARIELA PÉREZ CHAVARRÍA

\section{Conclusiones generales}

1. El estilo de comunicación de los empleados mexicanos es mucho más abierto y expresivo (activo, dinámico) que el de los finlandeses con respecto a la frecuencia de conductas comunicativas que usan cuando se comunican con sus jefes. En efecto, los empleados mexicanos usaron más frecuencia de conductas comunicativas en 7 de las 13 dimensiones del cuestionario. Estas áreas fueron las siguientes: Comunicación No Verbal (Dimensión 02), Comunicación Verbal (Dimensión 03), Comunicación Formal-Informal (Dimensión 04), Apertura Comunicativa (Dimensión 08), Uso de Canales de Comunicación (Dimensión 09), y Comunicación Relacionada con la Toma de Decisiones (Dimensión 12). (Ver los resultados estadísticos en la sección de Resultados).

2. El estilo de comunicación de los empleados finlandeses es más pasivo, reservado y menos expresivo que el de los mexicanos con respecto a la frecuencia de conductas comunicativas que usan cuando se comunican con sus jefes. En efecto, los empleados finlandeses exhibieron más frecuencia de conductas comunicativas que los empleados mexicanos en solamente 3 de las 13 dimensiones del cuestionario. Estas dimensiones fueron las siguientes: Habilidades para Escuchar (Dimensión 01), Dar Feedback (Dimensión 11), y Características Culturales y Comunicación (adaptar estilo de comunicación de acuerdo con las características culturales de los jefes) (Dimensión 13).

\section{Explicación cultural}

No es de extrañarse que el estilo de comunicación en la cultura mexicana tienda a ser más expresivo y abierto que el de la cultura finlandesa. La cultura mexicana se clasifica dentro de las culturas de contexto alto (HALL, 1959), la gente tiende a tocarse al saludar y las expresiones de afecto son abiertas. Incluso, entre superior- subordinado si hay suficiente confianza y cercanía, el saludo puede incluir desde un apretón de manos, hasta un abrazo, una palmada en la espalda o un beso en la mejilla tratándose de mujeres especialmente. También es posible que además de temas del trabajo se aborden asuntos familiares o personales, sobre todo por parte del empleado hacia su jefe. Se prefiere la comunicación cara a cara, a la comunicación mediada ya sea por teléfono, correo electrónico, o escrita. El estilo tiende a ser indirecto, es decir se dan rodeos al hablar porque se evita decir las cosas directamente. Incluso, la cortesía puede ser más importante que la verdad. Por otra parte, no hay que olvidar que en la muestra mexicana los encuestados fueron más mujeres que hombres, también hubo más superiores mujeres y estos factores seguramente incidieron en los resultados. Muller y Rowell (1997) dicen que las mujeres en posiciones directivas tienden a mantener la armonía en las relaciones con sus subordinados, hablan extensamente, su comunicación es abierta, dan apoyo y ayuda a los empleados, discuten los problemas y mantienen un alto nivel afectivo, con énfasis en las relaciones. 
En comparación con la comunicación mexicana, la comunicación finlandesa es menos expresiva. La cultura finlandesa se clasifica dentro de las culturas de contexto bajo (HALL, 1959). El silencio forma parte importante de la comunicación finlandesa. Para los finlandeses el silencio no es algo pasivo; por el contrario, tratan de recibir la información de la otra parte con quien se comunican y también de reducir la incertidumbre a través de la observación (SAJAVAARA \& LEHTONEN, 1997). En la cultura finlandesa, el buen comunicador es alguien que sabe expresarse de una forma corta y eficaz. La comunicación finlandesa es en general orientada al receptor, es decir, que la responsabilidad en el éxito de la comunicación está más en el que la recibe que en el que la envía. El finlandés trata de evitar ser primero en hablar y enfatiza el escuchar. (SALLINENKUPARINEN, 1987). El clima es un factor que tiene que ver bastante con la poca expresividad en la comunicación finlandesa. En Finlandia el invierno es bastante largo, frío y hay muy poca luz durante el día. Este factor climático ejerce gran influencia en la comunicación y en cierto tipo de comportamiento como el retirarse a la soledad. Los finlandeses son más expresivos durante los veranos, cuando hay sol durante las 24 horas, hay más calor y la gente suele estar más en la calle. Es interesante notar que la muestra para esta investigación se recogió en invierno, por lo tanto es posible que el clima haya influido en los resultados. Por otra parte, la comunicación finlandesa, tanto verbal como escrita, es directa, se expresa abiertamente lo que se quiere decir.

\section{Implicaciones teóricas y prácticas}

Concluimos este artículo con un breve análisis de las limitaciones de la investigación y de la importancia de la competencia comunicativa intercultural en las empresas y organizaciones.

\section{Limitaciones de la investigación}

Aunque las diferencias reportadas entre empleados finlandeses y mexicanos son estadísticamente significativas basadas en las muestras recogidas, sin embargo tenemos que ser cautelosos a la hora de generalizar estos resultados a otras empresas u organizaciones finlandesas y mexicanas con características culturales diferentes. Los resultados de esta investigación, como ya lo hemos indicado, están sin duda influenciados por características culturales específicas de las empresas y organizaciones en las que se recogieron las muestras. Por otra parte, las diferencias culturales entre las organizaciones que se comparan en este estudio son muy marcadas. Esta es sin duda una de las mayores limitaciones de este estudio. Por lo tanto, más estudios son necesarios donde se comparen organizaciones que sean más similares en cuanto a sus culturas, misión, tipo de gerencia, etc. Los autores creemos que como dicen Jones, Watson, Gardner, and Gallois (2004) el contexto dentro del cual ocurre la comunicación, tanto en el nivel local como global, debe ser explorado exhaustivamente si es que queremos entender completamente la comunicación en las organizaciones. 
DIFERENCIAS EN EL ESTILO DE COMUNICACIÓN ENTRE EMPLEADOS FINLANDESES Y MEXICANOS CUANDO SE COMUNICAN CON SUS JEFES: UN ESTUDIO COMPARATIVO • FEDERICO VARONA, PÄIVI VAAHTERIKKO-MEJÍA E MARIELA PÉREZ CHAVARRÍA

\section{Competencia comunicativa intercultural en las empresas}

El crecimiento de la diversidad étnica y cultural en las organizaciones y empresas a nivel mundial, debido al fenómeno de la migración y de la globalización, exige un tipo totalmente nuevo de de jefe (supervisor, gerente o superior). El nuevo tipo de jefe que necesitan tanto las organizaciones nacionales como internacionales es alguien que sea competente en comunicación intercultural; es decir, alguien que posea las siguientes características: Primero, conocimiento de su identidad cultural y de su estilo de comunicación. Segundo, conocimiento de la identidad cultural y del estilo de comunicación de sus empleados. Tercero, valorar la diversidad cultural y comunicativa del personal como un enorme recurso positivo para el éxito de la empresa u organización en esta era de la globalización. Cuarto, habilidades interculturales comunicativas para relacionarse con personas de diferentes culturas y estilos de comunicación. Sólo a través de la educación y el entrenamiento se pueden preparar a este nuevo tipo de jefes. Este entrenamiento tiene centrarse en la educación de valores, comportamientos, y conocimiento general y específico sobre las diferentes culturas y estilos de comunicación. Este es el gran reto que hoy tienen las organizaciones y empresas, ofrecer este tipo de educación y entrenamiento a todos sus cuadros de mando. Actualmente son contadas las empresas y organizaciones que lo hacen y que por lo tanto saben beneficiarse del conocimiento y de las habilidades culturales y comunicativas de sus empleados. Nos estamos refiriendo aquí a saber aprovechar el capital social (capacidades culturales y sociales de las personas, redes creadas y desarrolladas, y el conocimiento teórico y práctico que poseen los miembros de la organización con respecto, por ejemplo, a la forma de comunicarse y trabajar con gente de diferentes culturas, VAAHTERIKKO-MEJÍA, 2007). Pero en muchos casos, las organizaciones ni siquiera se interesan por el rico "bagaje intercultural" que tienen sus empleados y superiores, y mucho menos saben usarlo para el desarrollo de la organización.

El conocimiento por parte de jefes y superiores de las características del estilo de comunicación y de las características culturales de sus empleados son esenciales para crear y mantener una buena y exitosa comunicación y relación tanto en organizaciones nacionales como internacionales. Definitivamente, como lo revela el presente estudio, existen diferencias muy bien marcadas en el estilo de comunicación de empleados con sus superiores entre diferentes países, como el caso de Finlandia y México.

\section{Referencias}

Business Negotiations Finnish Negotiating with Latin-Americans. Article founded by Finnish Academy's SoCa programme. Disponível en: http://www.jyu.fi/erillis/agoracenter/en/research/soca/projects/ojala

CHEN, G.M. \& CHUNG, J. The impact of Confucianism on organizational communication. Communication Quarterly, 42, 93-105. 1994. 
DIFERENCIAS EN EL ESTILO DE COMUNICACIÓN ENTRE EMPLEADOS FINLANDESES Y MEXICANOS CUANDO SE COMUNICAN CON SUS JEFES: UN ESTUDIO COMPARATIVO - FEDERICO VARONA, PÄIVI VAAHTERIKKO-MEJÍA E MARIELA PÉREZ CHAVARRÍA

CONDON, J. C. Good neighbors: Communicating with the Mexicans. Intercultural Press Inc. 1985.

COX, T., Jr. Cultural diversity in organizations. San Francisco: Berrett-Koehler Publisher. 1993.

HALL, E. T. The silent language. New York: Doubleday \& Company, Inc. 1959.

HARRIS, Ph. R. \& MORAN, R. T. Managing cultural differences. (4th ed.). Houston, TX: Gulf Publishing Company. 1987.

HECHT, M. The conceptualization and measurement of interpersonal communication and satisfaction. Human Communication Research, 4, 253-264. 1978.

HOFSTEDE, G. Culture's consequences. (abridged edition). Newbury Park, CA: Sage Publications, 1984. Cultures and organizations: Software of the mind. London: McGraw-Hill Book Co., 1991. Cultural's consequences. Internal differences in work-related values. Beverly Hill: Sage Publications, 1980.

JONES, E., Watson, B., Gardner, J., \& Gallois, C., (2004). Organizational Communication: Challenges for the new century. Journal of Communication, 4, 722-750. 2004.

KRAS, E. S. Management in two cultures. Bridging the gap between U.S. and Mexican managers. Intercultural Press, Inc., 1988.

KREPS, G. L. \& KUNIMOTO, E. N. Effective communication in multicultural health care settings. Thousand Oaks, CA: Sage Publications, 1994.

MARTÍNEZ, S. \& DORFMAN, P. The Mexican entrepreneur: An ethnographic study of the Mexican empresario. International Studies of Management \& Organization, 2, 97-124. 1998.

NICHOLLS, Ch., LANE, H. \& BREHM, M. Taking self-managed teams to México. The Academy of Management Executive, 3. 15-25. 1999.

NORTON, R. Foundation of a communicator style construct. Human Communication Research, 4, 99-112. 1978. Communicator Style: Theory, Applications, and Measures. Newbury Park, A: Sage Publications, 1983.

MULLER y ROWELL Mexican women managers: an emerging profile. Human Resource Management. Winter 1997, Vol 36, No. 4. pp 423-435. 1997.

PALACIOS, L. Construcción de una cultura hegemónica en la gran industria regiomontana. 918-1954. 1999. Disponible en: http://mezcal.colmex.mx/historiadores/ponencias/162.pdf

SAJAVAARA, K. \& LEHTONEN, J. The silent Finn revisited. In: JAWORSKI, A. Silence: interdisciplinary perspectives. Berlin: Mouton Gruyter, 1997.

SALLINEN-KUPARINEN, A. Culture and communication image. Paper presented at the Western Speech Communication Association Convention: Salt Lake City, 1987. 
DIFERENCIAS EN EL ESTILO DE COMUNICACIÓN ENTRE EMPLEADOS FINLANDESES Y MEXICANOS CUANDO SE COMUNICAN CON SUS JEFES: UN ESTUDIO COMPARATIVO - FEDERICO VARONA, PÄIVI VAAHTERIKKO-MEJÍA E MARIELA PÉREZ CHAVARRÍA

SHUTER, R. \& WISEMAN, R. L. Communication in multicultural organizations: Conceptual, theoretical, and practical issues. In: WISEMAN, R. L. \& SHUTER, R. (Eds.). Communicating in multicultural organizations (pp. 3-11). Thousand Oaks, CA: Sage Publications, 1994.

TEBOUL, JC. B., CHEN, L., \& FRITZ, L. M. Intercultural organizational communication research in multinational organizations. In: WISEMAN, R. L. \& SHUTER, R. (eds.). Communicating in multicultural organizations (pp. 12-29). Thousand Oaks, CA: Sage Publications, 1994.

TROMPENAARS, F. and HAMPDEN-TURNER, C. Riding the Waves of Culture: Understanding Diversity in Global Business. New York: McGraw-Hill, 1998.

VAAHTERIKKO-MEJÍA, P. Cultural differences in Ibero-Nordic communication: perceptions about Finnish and Colombian negotiators. Jyväskylä: University of Jyväskylä. 2001. Disponível en: http://selene.lib.jyu.fi:8080/vaitos/studies/studcomm/9513914224.pdf

The Meaning of Intangibles in International

VARONA, F. Similarities and differences on employee/supervisor communication styles between Hispanics and Euroamericans. Paper Presented at the Speech Communication Association Convention. San Antonio, Texas, November 1995.

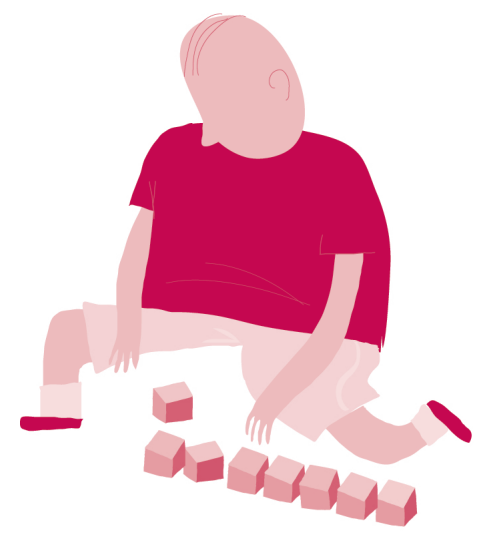

